\title{
Monitoring the Coefficient of Variation using Variable Sampling Interval CUSUM control charts
}

\author{
immediate
}

November 9, 2020

\begin{abstract}
In monitoring the coefficient of variation (CV), the variable sampling interval (VSI) type charts are a useful adaptive strategy that has been recently combined with various types of charts, including exponentially weighted moving average (EWMA) and variable sample size (VSS) charts. These combinations lead to desirable control charts with an impressive performance in detecting process shifts or anomalies. However, in these studies, the authors asserted that adaptive type charts are not competitive to monitor downward shifts in the CV. Therefore, they willingly omitted the decrease in process shifts. In this paper, we suggest overcoming this downside by designing two one-sided VSI CUSUM control charts for tracking the CV. The performance of the proposed charts is evaluated by the average time to signal (ATS) criterion. The numerical results show that our proposed adaptive type charts are efficient charts to monitor downward shifts in the CV.
\end{abstract}

Keywords: VSI, CUSUM, Coefficient of Variation, Markov Chain, Anomaly Detection.

\section{Introduction}

The control chart is a useful tool that is widely used in Statistical Process Monitoring (SPM) to detect changes in underlying processes. Recently, the need to detect early abnormalities has been raised. Numerous fields using the control chart approach for anomaly detection have received great attention from the science community: emergency hospital systems ${ }^{9}$, traffic measurement ${ }^{12}$, etc. Moreover, various techniques based on control charts and machine learning methods have also been developed to investigate this issue $^{15}$.

A coefficient of variation $(\mathrm{CV})$ is a statistical measure of the relative dispersion of a variable to its mean. In many practical processes, the CV is much 
more important than the mean and the variance: the processes are still considered to be in-control regardless of how their means and variances change from sample to sample, as long as their CVs remain constant. The applications of the CV can be seen in a large number of fields such as manufacturing industries, finance, and health sciences; see, for example, Castagliola et al. ${ }^{7}$ and Yeong et al. ${ }^{19}$ for more discussions about these applications. Then it is worth monitoring the CV in many quality control problems. In the year 2007, Kang et al. ${ }^{10}$ firstly suggested a Shewhart control chart for monitoring the CV. Although having an advantage of an easy-to-design control chart, the Shewhart chart is well-known to be inefficient in detecting small or moderate process shifts. For this reason, many other control schemes have been proposed. Castagliola et al. ${ }^{7}$ used the exponentially weighted moving average (EWMA) type chart for monitoring the CV squared and applied this chart to track the pressure drop time related to the pore shrinkage in a metal sintering process. A synthetic type chart and a cumulative sum (CUSUM) type chart for monitoring the CV are investigated by Calzada and Scariano ${ }^{4}$ and Tran and Tran ${ }^{16}$, respectively.

There are also other approaches to enhance the efficiency of control charts where the sampling interval or the sample size are enabled to vary from sample to sample. Varying the sampling interval or sample size allows us to obtain more or faster information about the process shifts when they do exist, thus improving the performance of the chart in detecting assignable causes. Recently, these adaptive strategies have already been considered in monitoring the CV. Castagliola et al. ${ }^{6}$ designed the VSS control charts for monitoring a transformed statistic of the CV, which approximately follows a standard normal distribution. Amdouni et al. ${ }^{1}$ proposed the same type of VSS control charts to directly monitor the CV while Khaw et al. ${ }^{11}$ combined both VSI and VSS strategies in a Shewart chart. Tracking the CV is also done by incorporating VSI into the EWMA chart according to the suggestion of Yeong et al. ${ }^{18}$ and Tran et al. ${ }^{17}$. The authors of the last two aforementioned papers showed that their proposed control charts are actually more effective than others such as Shewhart chart, synthetic chart, EWMA chart, or VSI Shewhart chart. In these papers, however, the authors have only considered upward shifts arguing that adaptive type charts are not competitive to monitor downward shifts in the CV, see Castagliola et al. ${ }^{5}$. That is to say, the authors willingly omitted the situations corresponding to the decrease process shifts. This does not meet the practical requirements as in many cases, the quality practitioners wish to track the reduction of the process. The purpose of this paper, therefore, is to design a control chart to fill this gap. We suggest to integrate VSI into the CUSUM type chart, which is proved to have attractive performance in detecting small shifts, see $^{16}$. By incorporating these two chart features, the resulting control chart is expected not only to detect upward but also downward sides of process 
shifts. Because monitoring $S^{2}$ using CUSUM chart is often more efficient than monitoring $S$ or any function of $S$, where $S$ denotes the statistical interest $\left(\mathrm{see}^{7}\right.$ ), we will use the VSI CUSUM to monitor the CV squared $\gamma^{2}$ rather than the CV $\gamma$ itself. We call our control chart the VSI CUSUM- $\gamma^{2}$ control chart.

The remainder of our paper is organized as follows. In Section 2, we recall a brief review of the distribution of the sample coefficient of variation. Two one-sided VSI CUSUM- $\gamma^{2}$ control charts for monitoring the CV are defined in Section 3. Section 4 is devoted to calculating the average time to signal $A T S$, a measure to evaluate the performance of the proposed charts. Section 5 provides the optimal design parameters for different scenarios and for a wide range of deterministic shifts, including both decreasing and increasing CV shifts. An example illustrates the use of VSI CUSUM- $\gamma^{2}$ charts on real data in Section 6. Some remarks and conclusions are given in Section 7.

\section{The distribution of the sample CV}

In this section, we present a brief review of the distribution of the sample coefficient of variation. Being defined as the ratio of the standard deviation $\sigma$ to the mean $\mu$ of a random variable $X$, the CV of $X$ is

$$
\gamma=\frac{\sigma}{\mu} .
$$

Let $\bar{X}$ and $S$ be the sample mean and the sample standard deviation of $X_{1}, \ldots, X_{n}$, where observed subgroups $\left\{X_{1}, \ldots, X_{n}\right\}$ are $n$ independently and identically distributed normal random variables with mean $\mu$ and standard deviation $\sigma$. That is

$$
\bar{X}=\frac{1}{n} \sum_{i=1}^{n} X_{i}
$$

and

$$
S=\sqrt{\frac{1}{n-1} \sum_{i=1}^{n}\left(X_{i}-\bar{X}\right)^{2}} .
$$

By definition, the sample coefficient of variation $\hat{\gamma}$ is defined as

$$
\hat{\gamma}=\frac{S}{\bar{X}}
$$

We will describe below the calculation steps to obtain the c.d.f function of this quantity. By definition, $\hat{\gamma}^{2}$ is defined on $(0,+\infty)$. From the result of ${ }^{8 ; 7}$, we noted that $\frac{n}{\hat{\gamma}^{2}}$ follows a noncentral $F$ distribution with $(1, n-1)$ degrees of freedom and noncentrality parameter $\frac{n}{\gamma^{2}}$. Based on this property, it is 
easy to derive the exact c.d.f. (cumulative distribution function) $F_{\hat{\gamma}^{2}}(x \mid n, \gamma)$ of $\hat{\gamma}^{2}$ as

$$
\begin{aligned}
F_{\hat{\gamma}^{2}}(x \mid n, \gamma) & =P\left(\hat{\gamma}^{2} \leqslant x \mid n, \gamma\right) \\
& =P\left(\frac{n}{\hat{\gamma}^{2}} \geqslant \frac{n}{x}>0 \mid n, \gamma\right) \\
& =1-P\left(0<\frac{n}{\hat{\gamma}^{2}} \leqslant \frac{n}{x} \mid n, \gamma\right) \\
& =1-F_{F}\left(\frac{n}{x} \mid 1, n-1, \frac{n}{\gamma^{2}}\right),
\end{aligned}
$$

where $F_{F}\left(. \mid 1, n-1, \frac{n}{\gamma^{2}}\right)$ is the c.d.f. of the noncentral $F$ distribution.

Similarly, the p.d.f of $\hat{\gamma}^{2}$ is

$$
f_{\hat{\gamma}^{2}}(x \mid n, \gamma)=\frac{n}{x^{2}} f_{F}\left(\frac{n}{x} \mid 1, n-1, \frac{n}{\gamma^{2}}\right),
$$

where $f_{F}\left(. \mid 1, n-1, \frac{n}{\gamma^{2}}\right)$ is the p.d.f of the noncentral $F$ distribution. Figure 1 presents the density function of $\hat{\gamma}^{2}$ for different parameters. The inverse c.d.f. of $\hat{\gamma}^{2}$ is deduced by inverting its c.d.f., resulting in

$$
F_{\hat{\gamma}^{2}}^{-1}(\alpha \mid n, \gamma)=\frac{n}{F_{F}^{-1}\left(1-\alpha \mid 1, n-1, \frac{n}{\gamma^{2}}\right)},
$$

where $F_{F}^{-1}\left(. \mid 1, n-1, \frac{n}{\gamma^{2}}\right)$ is the inverse c.d.f. of the noncentral $F$ distribution.

\section{Implementation of VSI CUSUM- $\gamma^{2}$ control charts}

Assume that we observe a subgroup of $n$ independently and identically distributed normal random variables $\left\{X_{i, 1}, X_{i, 2}, \ldots, X_{i, n}\right\}$ at time $i=1,2, \ldots$, in which each random variable $X_{i, j}$ follows a normal distribution with parameters $\left(\mu_{i}, \sigma_{i}\right)$. When the process is in-control, parameters $\mu_{i}$ and $\sigma_{i}$ are supposed to be constrained by the relation $\gamma_{i}=\frac{\sigma_{i}}{\mu_{i}}=\gamma_{0}$. This means from one subgroup to another, the values of $\mu_{i}$ and $\sigma_{i}$ might fluctuate, but the coefficient of variation $\gamma_{i}=\frac{\sigma_{i}}{\mu_{i}}$ remains constant, being equal to some predefined in-control values $\gamma_{0}$, common to all the subgroups, see ${ }^{7}$. If the in-control value $\gamma_{0}$ is unknown, it has to be estimated from a Phase I data set, composed of $k=1, \ldots, m$ subgroups $\left\{X_{i, 1}, \ldots, X_{i, n}\right\}$ of size $n$. Let us assume that there is independence within and between subgroups, we 


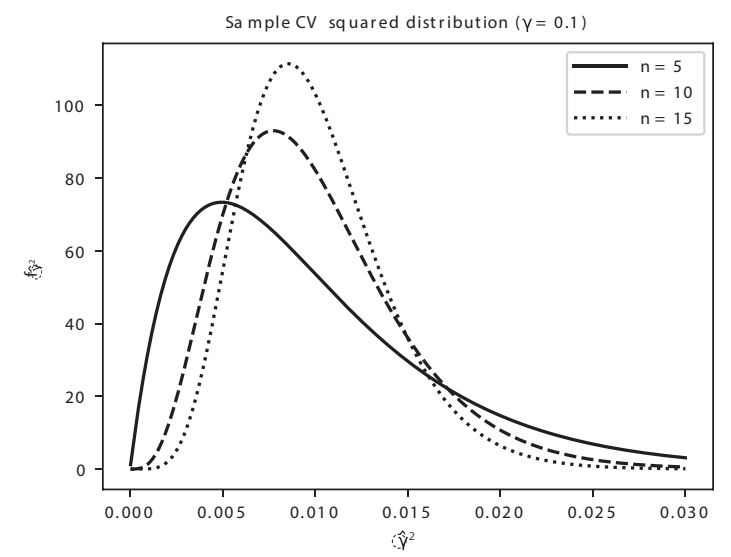

Figure 1: Density function of sample $\gamma^{2}$

could get the estimator $\hat{\gamma}_{0}$ for $\gamma_{0}$ by using a root-mean-square computation Castagliola et al. ${ }^{7}$. The estimator $\hat{\gamma}_{0}$ for $\gamma_{0}$ is

$$
\hat{\gamma}_{0}=\sqrt{\frac{1}{m} \sum_{k=1}^{m} \gamma_{k}^{2}}
$$

where $\hat{\gamma}_{k}$ is the sample coefficient of variation of subgroup $k$, see Castagliola et al. ${ }^{7}$ for more details.

There are no closed-form of $\mu_{0}\left(\hat{\gamma}^{2}\right)$ and $\sigma_{0}\left(\hat{\gamma}^{2}\right)$, the in-control mean and standard deviation of $\hat{\gamma}^{2}$, but accurate approximations are provided by Breunig $^{2}$. They are

$$
\begin{aligned}
\mu_{0}\left(\hat{\gamma}^{2}\right) & =\gamma_{0}^{2}\left(1-\frac{3 \gamma_{0}^{2}}{n}\right), \text { and } \\
\sigma_{0}\left(\hat{\gamma}^{2}\right) & =\sqrt{\gamma_{0}^{4}\left(\frac{2}{n-1}+\gamma_{0}^{2}\left(\frac{4}{n}+\frac{20}{n(n-1)}+\frac{75 \gamma_{0}^{2}}{n^{2}}\right)\right)-\left(\mu_{0}\left(\hat{\gamma}^{2}\right)-\gamma_{0}^{2}\right)^{2}} .
\end{aligned}
$$

Tran and Tran ${ }^{16}$ suggested the following two separate one-sided CUSUM charts for monitoring CV squared:

- an upward CUSUM chart to detect an increase in the CV, defined by

$$
C_{i}^{+}=\max \left(0, C_{i-1}^{+}+\left(\hat{\gamma}_{i}^{2}-\mu_{0}\left(\hat{\gamma}^{2}\right)\right)-K^{+}\right)
$$

with the initial value $C_{0}^{+}=0$ and the corresponding upper control limit $U C L^{+}=H_{U} \times \mu_{0}\left(\hat{\gamma}^{2}\right)>0$, 
- a downward CUSUM chart to detect a decrease in the CV, defined by

$$
C_{i}^{-}=\max \left(0, C_{i-1}^{-}-\left(\hat{\gamma}_{i}^{2}-\mu_{0}\left(\hat{\gamma}^{2}\right)\right)-K^{-}\right)
$$

with the initial value $C_{0}^{-}=0$ and the corresponding lower control limit $U C L^{-}=H_{D} \times \mu_{0}\left(\hat{\gamma}^{2}\right)>0$,

The reference parameters can be written in the forms: $K^{+}=K_{U} \times \sigma_{0}\left(\hat{\gamma}^{2}\right)$ $\left(K^{-}=K_{D} \times \sigma_{0}\left(\hat{\gamma}^{2}\right)\right)$, where $K_{U}$ and $K_{D}$ are the charts parameters needed to be found.

The above CUSUM- $\gamma^{2}$ control charts are designed with fixed sampling intervals (FSI). In VSI type charts, the control limits are still kept the same as in FSI type charts; however, the sampling intervals are enabled to vary. That is to say, the time between two successive samples $\hat{\gamma}_{i+1}^{2}$ and $\hat{\gamma}_{i}^{2}$ is determined on the basis of the current value of $C_{i}^{+}\left(C_{i}^{-}\right)$. This is performed by introducing new warning limits defined as

- for the upward VSI CUSUM- $\gamma^{2}$ control chart,

$$
U W L^{+}=R_{U} \times U C L^{+},
$$

- for the downward VSI CUSUM- $\gamma^{2}$ control chart,

$$
U W L^{-}=R_{D} \times U C L^{-},
$$

where $R_{U} \in(0,1)\left(R_{D} \in(0,1)\right)$ is called the warning limit coefficient. With this new parameter, the control interval is separated into three regions: the safe region $\left(\left(0, U W L^{-}\right]\right.$or $\left.\left(0, U W L^{+}\right]\right)$, the warning region $\left(\left(U W L^{-}, U C L^{-}\right]\right.$ or $\left.\left(U W L^{+}, U C L^{+}\right]\right)$and the out-of-control region (otherwise). The values of $R_{U}\left(R_{D}\right)$ determine the width of the warning region: the closer to 0 the warning limit coefficient, the larger the warning region compared to the control interval. With this subdivision, the VSI strategy works as follows:

- If the current sample $C_{i}^{+}\left(C_{i}^{-}\right)$falls into the safe region, the process is considered to be "in-control" and the next sample is selected after a longer interval, say $h_{L}$.

- If current sample $C_{i}^{+}\left(C_{i}^{-}\right)$is observed in the warning region, the process might be "at risk", the next sampling interval should be shorter, say $h_{S}$, to quickly collect more information and detect assignable causes if there are any.

- Otherwise, the process is considered to be out-of-control and actions should be taken to locate and remove assignable causes.

Figure 2 illustrates a graphical view about the operation rules of the upward VSI CUSUM- $\gamma^{2}$ chart. 


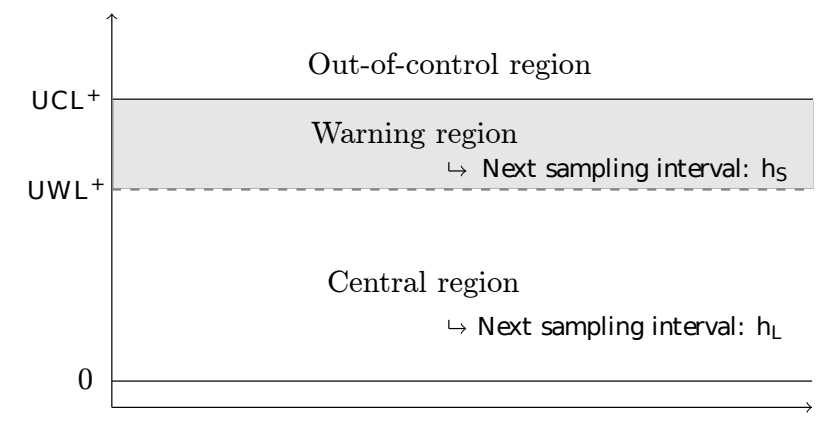

Figure 2: Three regions and operation rules of the upward VSI CUSUM- $\gamma^{2}$ control chart.

\section{Design of optimal VSI CUSUM- $\gamma^{2}$ control charts}

We evaluate the performance of the proposed charts by using the average time to signal $(A T S)$, which is a statistical measure representing the expected time before a control chart signals an "out-of-control" condition. Once the control chart parameters are defined, the ATS can be numerically computed as the process has been shifted from an in-control value $\gamma_{0}$ to an out-of-control value $\gamma_{1}=\tau \gamma_{0}$, where $\tau$ is the shift size. The value $\tau<1$ corresponds to a decrease of the nominal $\mathrm{CV}$, while the value $\tau>1$ is corresponding to its increase. Let $A T S_{0}$ denote the in-control value and $A T S_{1}$ denote the out-of-control value of $A T S$. We will use the Markov chain approach to calculate the ATS for VSI CUSUM- $\gamma^{2}$ charts. Firstly, the control interval is partitioned into a finite set of $p$ sub-intervals corresponding to $p+1$ states of the Markov chain. The width of the first sub-interval is $\delta=\frac{U C L^{+}}{2 p-1}\left(\delta=\frac{U C L^{-}}{2 p-1}\right)$ and the width of the others is $2 \delta$. Figure 3 demonstrates this subdivision for the upward control chart.

In Figure 3, the first sub-interval corresponds to the state 0; each other sub-interval, say $\left(H_{j}-\delta, H_{j}+\delta\right]$, represents the in-control transient state $j$ of a Markov chain, $j=1, . ., p-1$ while the state $p$ is the out-of-control or absorbing state. If the statistic $C_{i}^{+}\left(C_{i}^{-}\right)$falls into the sub-interval $j$, the Markov chain is in the transient state $j, j=0,1, . ., p-1$, for sample $i$; if not, the chain reaches the absorbing state. The value of $p$ is chosen large enough so that the midpoint $H_{j}$ can be considered as an approximately representative value of the statistic and this finite approach provides an accurate approximation of the $A T S$. The transition probability matrix $\mathbf{P}$ 


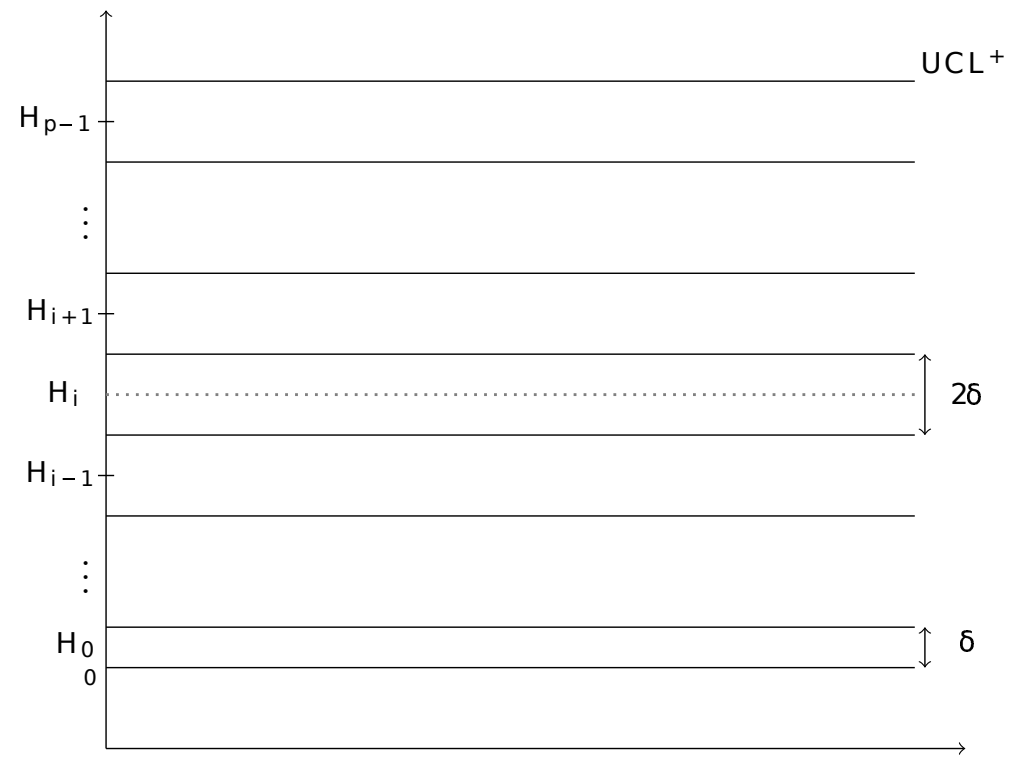

Figure 3: $p$ sub-intervals of control interval: the first sub-interval is $\delta$ in width, the others are $2 \delta$ in width.

of this Markov chain is

$$
\mathbf{P}=\left(\begin{array}{cc}
\mathbf{Q} & \mathbf{r} \\
\mathbf{0}^{T} & 1
\end{array}\right)=\left(\begin{array}{ccccc}
Q_{0,0} & Q_{0,1} & \ldots & Q_{0, p-1} & r_{0} \\
Q_{1,0} & Q_{1,1} & \ldots & Q_{1, p-1} & r_{1} \\
\vdots & \vdots & & & \vdots \\
Q_{p-1,0} & Q_{p-1,1} & \ldots & Q_{p-1, p-1} & r_{p-1} \\
0 & 0 & \ldots & 0 & 1
\end{array}\right)
$$

where $\mathbf{Q}$ is the $(p, p)$ matrix of transient probabilities, $\mathbf{0}=(0,0, \ldots, 0)^{T}$ and $p$-vector $\mathbf{r}=(\mathbf{1}-\mathbf{Q} \mathbf{1})$ with $\mathbf{1}=(1,1, \ldots, 1)^{T}$. The representative element $Q_{i, j}, i=0,1, \ldots, p-1$, of the matrix $\mathbf{Q}$ is equal to

- for the upward chart,

$$
\begin{aligned}
Q_{i, 0}= & F_{\hat{\gamma}^{2}}\left(\mu_{0}\left(\hat{\gamma}^{2}\right)-H_{i}+K^{+}+\delta \mid n, \gamma_{1}\right), \\
Q_{i, j}= & F_{\hat{\gamma}^{2}}\left(\mu_{0}\left(\hat{\gamma}^{2}\right)+H_{j}-H_{i}+\delta+K^{+} \mid n, \gamma_{1}\right) \\
& -F_{\hat{\gamma}^{2}}\left(\mu_{0}\left(\hat{\gamma}^{2}\right)+H_{j}-H_{i}-\delta+K^{+} \mid n, \gamma_{1}\right) ;
\end{aligned}
$$

- for the downward chart,

$$
\begin{aligned}
Q_{i, 0}= & 1-F_{\hat{\gamma}^{2}}\left(\mu_{0}\left(\hat{\gamma}^{2}\right)+H_{i}-K^{-}-\delta \mid n, \gamma_{1}\right), \\
Q_{i, j}= & F_{\hat{\gamma}^{2}}\left(\mu_{0}\left(\hat{\gamma}^{2}\right)+H_{j}-H_{i}+\delta+K^{+} \mid n, \gamma_{1}\right) \\
& -F_{\hat{\gamma}^{2}}\left(\mu_{0}\left(\hat{\gamma}^{2}\right)+H_{j}-H_{i}-\delta+K^{+} \mid n, \gamma_{1}\right),
\end{aligned}
$$


where $F_{\hat{\gamma}^{2}}($.$) is the c.d.f of \hat{\gamma}^{2}$ as defined in (??). Consider the zero-state performance, the vector $\mathbf{q}$ of initial probability is set equal to $\mathbf{q}=(1,0, \ldots, 0)^{T}$, i.e., the initial state corresponds to the "restart state".

The ATS now can be computed by the following formula as in Saccucci et al. ${ }^{14}$ :

$$
A T S=\mathbf{q}^{T}(\mathbf{I}-\mathbf{Q})^{-1} \mathbf{g}
$$

where $\mathbf{I}$ is the identity matrix, $\mathbf{g}$ is the vector of sampling intervals corresponding to the discretized states of the Markov chain. The element $g_{j}$ of the vector $\mathbf{g}$ is the sampling interval when the control statistic is in the state $j$ (denoted by $H_{j}$ ). It can be expressed by

$$
g_{j}=\left\{\begin{array}{ll}
h_{L} & \text { if } H_{j} \text { is in safe region } \\
h_{S} & \text { if } H_{j} \text { is in warning region }
\end{array} .\right.
$$

Commonly, a measure of average run length $A R L$ is used to evaluate the performance of FSI type charts. Due to fixed sampling interval, it is easy to convert the $A R L$ to $A T S$ in FSI version by the formula $A T S^{F S I}=$ $h_{F} \times A R L$, where $h_{F}$ stands for a constant sampling interval. In VSI version, the multiple $h_{F}$ must be converted to $E(h)$, the average sampling interval, that is

$$
A T S^{V S I}=E(h) \times A R L .
$$

The $A R L$ in (16) is calculated by, see Brook and Evans ${ }^{3}$,

$$
A R L=\mathbf{q}^{T}(\mathbf{I}-\mathbf{Q})^{-1} \mathbf{1}
$$

Equations (14), (16) and (17) lead to

$$
E(h)=\frac{\mathbf{q}^{T}(\mathbf{I}-\mathbf{Q})^{-1} \mathbf{g}}{\mathbf{q}^{T}(\mathbf{I}-\mathbf{Q})^{-1} \mathbf{1}}
$$

A fair comparison between both types of control charts is made by imposing the same in-control values of $A T S_{0}$ and $E_{0}(h)$ (the out-of-control values will be denoted by $A T S_{1}$ and $E_{1}(h)$ ). Without loss of generality, we can assume that $h_{F}=1$, leading to the constraint $E_{0}(h)=1$ in the VSI CUSUM- $\gamma^{2}$ control chart.

It is our desire to design a control chart with smaller $A T S_{1}$ and the same $A T S_{0}$ and $E_{0}(h)$ compared to other charts. We then wish to find out an optimal combination of the parameters of the VSI CUSUM- $\gamma^{2}$ charts which minimizes the out-of-control ATS subject to the constraint on $A T S_{0}$ and $E_{0}(h)$. In design procedure of the VSI CUSUM- $\gamma^{2}$ control charts, a fixed couple $\left(h_{S}, h_{L}\right)$ is usually used, see, for example, Reynolds and Arnold ${ }^{13}$, 
Castagliola et al. ${ }^{5}$ and Yeong et al. ${ }^{18}$. Predetermining the value of $h_{S}$ is reasonable because there is always a certain time interval between two products or two batches for any process. However, it is not really convincing to predetermine $h_{L}$. Our goal is to design a control chart with the best performance. Thus, we must focus on controlling the chart parameters rather than assuming process parameters. We handle this problem by setting up a relation between the control limit and the warning limit represented by the warning limit coefficients of $R_{U}$ and $R_{D}$. We will minimize the $A T S_{1}$ with respect to $\left(K_{U}^{*}, H_{U}^{*}, h_{L}^{*}\right)$ (upward chart) or $\left(K_{D}^{*}, H_{D}^{*}, h_{L}^{*}\right)$ (downward chart) using some small values of $h_{S}$. That is to say, for a specific value of shift size, we wish to find out the triple $\left(K_{U}^{*}, H_{U}^{*}, h_{L}^{*}\right)$ or $\left(K_{U}^{*}, H_{U}^{*}, h_{L}^{*}\right)$ such that

- for the downward chart,

$$
\left(K_{D}^{*}, H_{D}^{*}, h_{L}^{*}\right)=\underset{\left(K_{D}, H_{D}, h_{L}\right)}{\operatorname{argmin}} \operatorname{ATS}\left(n, K_{D}, H_{D}, R_{D}, \gamma_{0}, \tau, h_{L}, h_{S}\right)
$$

subject to the constraint

$$
\left\{\begin{array}{l}
A T S\left(n, K_{D}, H_{D}, R_{D}, \gamma_{0}, \tau=1, h_{L}, h_{S}\right)=A T S_{0} \\
E_{0}(h)=1
\end{array} ;\right.
$$

- for the upward chart,

$$
\left(K_{U}^{*}, H_{U}^{*}, h_{L}^{*}\right)=\underset{\left(K_{U}, H_{U}, h_{L}\right)}{\operatorname{argmin}} \operatorname{ATS}\left(n, K_{U}, H_{U}, R_{U}, \gamma_{0}, \tau, h_{L}, h_{S}\right)
$$

subject to the constraint

$$
\left\{\begin{array}{l}
A T S\left(n, K_{U}, H_{U}, R_{U}, \gamma_{0}, \tau=1, h_{L}, h_{S}\right)=A T S_{0} \\
E_{0}(h)=1
\end{array} .\right.
$$

The design procedure of VSI CUSUM- $\gamma^{2}$ charts is implemented by finding out the optimal triple $\left(K_{U}^{*}, H_{U}^{*}, h_{L}^{*}\right)$ (upward chart) or $\left(K_{D}^{*}, H_{D}^{*}, h_{L}^{*}\right)$ (downward chart) which minimizes the out-of-control ATS for a given incontrol $A T S=A T S_{0}$ and $E_{0}=1$. The choice of the optimal triple generally entails two steps:

1. Find the potential combinations $\left(K_{U}, H_{U}, h_{L}\right)$ (upward chart) or $\left(K_{D}\right.$, $H_{D}, h_{L}$ ) (downward chart) such that $A T S=A T S_{0}$ and $E_{0}=1$ (where $A T S_{0}$ is some predefined "in-control" $A T S$ value).

2. Choose, among these potential combinations $\left(K_{U}, H_{U}, h_{L}\right)$ (upward chart) or $\left(K_{D}, H_{D}, h_{L}\right)$ (downward chart), the one $\left(K_{U}^{*}, H_{U}^{*}, h_{L}^{*}\right)$ (upward chart) or $\left(K_{D}^{*}, H_{D}^{*}, h_{L}^{*}\right)$ (downward chart) allowing for the best performance, i.e., the smallest "out-of-control" $A T S$ value for a particular shift $\tau$, from an in-control value $\gamma_{0}$ to an out-of-control value $\gamma_{1}=\tau \gamma_{0}$. 
In this study, like in Tran and Tran ${ }^{16}$, in order to find these optimal triples $\left(K_{U}^{*}, H_{U}^{*}, h_{L}^{*}\right)$ (upward chart) or $\left(K_{D}^{*}, H_{D}^{*}, h_{L}^{*}\right)$ (downward chart), we simultaneously use a nonlinear equation solver together with an optimization algorithm through an algorithm developed in Scicoslab software. More specifically, for fixed values of $\gamma_{0}, n, h_{S}, R_{U}$ (upward chart) or $R_{D}$ (downward chart) and $\tau=1$ we search the optimal triple $\left(K_{U}^{*}, H_{U}^{*}, h_{L}^{*}\right)$ (upward chart) or $\left(K_{D}^{*}, H_{D}^{*}, h_{L}^{*}\right)$ (downward chart) such that $A T S\left(n, K_{D}, H_{D}, R_{D}, \gamma_{0}, \tau=\right.$ $\left.1, h_{L}, h_{S}\right)=A T S_{0}$ (downward chart) or $\operatorname{ATS}\left(n, K_{U}, H_{U}, R_{U}, \gamma_{0}, \tau=1, h_{L}, h_{S}\right)=$ $A T S_{0}$ (upward chart) and $E_{0}(h)=1$ (using the nonlinear equation solver), minimizing $A T S\left(n, K_{D}, H_{D}, R_{D}, \gamma_{0}, \tau, h_{L}, h_{S}\right)$ or $A T S\left(n, K_{U}, H_{U}, R_{U}, \gamma_{0}, \tau, h_{L}, h_{S}\right)$ (using the optimizer).

\section{Numerical results and comparison}

We explore in this section the statistical performance of the proposed control charts for various out-of-control situations. The optimal triples $\left(K_{D}^{*}, h_{D}^{*}, h_{L}\right)$ (downward chart) and $\left(K_{U}^{*}, h_{U}^{*}, h_{L}\right)$ (upward chart) minimizing $A T S_{1}$ are solved subject to the constraints $A T S_{0}=370.4$ and $E_{0}(h)=1$. We consider the following situations of parameters.

- $n \in\{5,7,10,15\}$.

- $\tau \in\{0.5,0.65,0.80,0.90,1.1,1.2,1.5,2.0\}$.

- $R_{D}\left(R_{U}\right) \in\{0.05,0.1,0.3,0.5,0.7\}$.

- $h_{S} \in\{0.1,0.5\}$.

- $\gamma_{0} \in\{0.05,0.10,0.15,0.20\}$.

Given the values of $\gamma_{0}, n, h_{S}$ and $R_{D}\left(R_{U}\right)$, the optimal triple $\left(K_{U}^{*}, H_{U}^{*}, h_{L}^{*}\right)$ in equation (19) for upward chart and $\left(K_{D}^{*}, H_{D}^{*}, h_{L}^{*}\right)$ in equation (21) for downward chart are solved for a particular shift size. The out-of-control $A T S_{1}$ are then numerically obtained. Tables 1-4 display the values of $A T S_{1}$ corresponding to optimal parameters $\left(K_{U}^{*}, H_{U}^{*}, h_{L}^{*}\right)$ or $\left(K_{D}^{*}, H_{D}^{*}, h_{L}^{*}\right)$ for the case $n=5,7,10$ and 15 . The first row of these tables corresponds to the value of $h_{S}$, the second row to the value of $R_{U}$ or $R_{D}$. The value of $\gamma_{0}$ is at the beginning of each block. For the comparison purpose, the values $\left(K_{U}, H_{U}, A R L_{1}\right)$ and $\left(K_{D}, H_{D}, A R L_{1}\right)$ of FSI CUMSUM- $\gamma^{2}$ corresponding to the same values of $n, \tau, \gamma_{0}$ are also shown in each table on the column entitled FSI. A number of general conclusions can be drawn from these tables as follows.

- In general, the VSI CUSUM- $\gamma^{2}$ control charts lead to better performance compared to the FSI CUSUM- $\gamma^{2}$ control charts. The values of 
$A T S_{1}$ are always smaller than the values of $A R L_{1}$; the difference between $A T S_{1}$ and $A R L_{1}$ is considerable, especially when the shift size is small. For example, in Table 1 with $n=5, \gamma_{0}=0.05, h_{S}=0.1, \tau=0.9$ and $R_{D}=0.1$, we have $A R L_{1}=50.9$, which is almost two times $A T S_{1}=26.4$. We can say that the VSI adaptive strategy remarkably improves the performance of CUSUM- $\gamma^{2}$ control charts in detecting small shifts.

- From Tables 1-4, an increase in the sample size $n$ reduces both the width of control limit represented by $H_{U}^{*}\left(H_{D}^{*}\right)$ and sampling interval $h_{L}^{*}$. For example, when $\gamma_{0}=0.1, \tau=1.1, h_{S}=0.5$ and $R_{U}=0.1$, we get $\left(H_{U}^{*}, h_{L}^{*}\right)=(5.60,1.58)$ for $n=7$ in Table 2 but $\left(H_{U}^{*}, h_{L}^{*}\right)=$ $(2.87,1.44)$ for $n=15$ in Table 4.

- Given the values of $n, \tau, R_{U}\left(R_{D}\right)$ and $\gamma_{0}$, the $A T S_{1}$ varies with $h_{S}$. In particular, an increase in $h_{S}$ makes the power of the VSI CUSUM$\gamma^{2}$ charts decrease. For instance, when $n=7, \gamma_{0}=0.2, \tau=1.2$ and $R_{U}=0.5$ in Table 2, we have $A T S_{1}=11.6$ for $h_{S}=0.1$ and $A T S_{1}=13.5$ for $h_{S}=0.5$.

- The in-control value $\gamma_{0}$ does not have much effect on the performance of VSI CUSUM- $\gamma^{2}$ control charts their control limits or sampling intervals. Given the values of the chart parameters, the value of $\left(H_{U}^{*}, h_{L}^{*}, A T S_{1}\right)$ and $\left(H_{U}^{*}, h_{L}^{*}, A T S_{1}\right)$ are either unchanged or changed insignificantly when $\gamma_{0}$ increases from 0.05 to 0.2 in every table.

- The predetermined value of $R_{U}$ or $R_{D}$ of the warning limit coefficient has a strong effect on the VSI CUSUM- $\gamma^{2}$ control charts. The smaller the predetermined value of $R_{U}$ or $R_{D}$ is, the smaller the value of $A T S_{1}$ is, and the larger the value of $h_{L}^{*}$ is. For example, in Table 1 when $n=5, \gamma_{0}=0.15, \tau=0.8$, one can see $A T S_{1}=8.5$ and $h_{L}^{*}=2.50$ for $R_{D}=0.05$ while $A T S_{1}=16.6$ and $h_{L}^{*}=1.02$ for $R_{D}=0.7$. This is because smaller values of $R_{U}\left(R_{D}\right)$ correspond to a larger portion of the warning region covering the in-control interval, or equivalently, a smaller safe region. As a result, the charts are more sensitive to the variability of the process, making the average time to detect an out-of-control condition shorter. However, one should know that too small values of this coefficient correspond to a very small central region. Consequently, most of the samples plotted in the charts will fall into the warning region and sub-sample will be taken more often. Moreover, by experimenting more with smaller values of $R_{U}\left(R_{D}\right)$, we find that there is an insignificant improvement when we reduce the value of $R_{U}\left(R_{D}\right)$ to less than 0.05 . For example, with $n=5, \tau=1.2, \gamma_{0}=0.05, h_{S}=0.1$ we have $A T S_{1}=10.44$ when $R_{U}=$ 0.03 and $A T S_{1}=10.42$ for $R_{U}=0.01$, compared to $A T S_{1}=10.5$ 
for $R_{U}=0.05$ as shown in Table 1 . The value of $R_{U}=0.05$ is then recommended to use in designing the VSI CUSUM- $\gamma^{2}$ charts.

- In general, the values of $A T S_{1}$ in the obtained results corresponding to the value of $\tau<1$ (downward chart) are relative small. Even, with the same distance of the shift size from the out-of-control condition to the in-control condition (i.e from the current value of $\tau$ to 1 ), the downward chart gives better performance in comparison with the upward chart. For example, with $n=10, R_{U}=R_{D}=0.1, h_{S}=0.1, \gamma_{0}=0.05$, we have $A T S_{1}=2.0$ when $\tau=1.5$ (upward chart) and $A T S_{1}=1.1$ when $\tau=0.5$ (downward chart). Thus, we can say that the downward VSI CUSUM- $\gamma^{2}$ chart is efficient in detecting decreasing shift sizes. This makes a correction to an argument that CV charts with varying charting parameters lead to poor performance when they are adopted to detect a decrease in the $\mathrm{CV}$ as in Castagliola et al. ${ }^{5}$; Yeong et al. ${ }^{18}$ and Khaw et al. ${ }^{11}$

The control charts designed above are based on the assumption that we are able to predict a deterministic shift size. This requirement seems to be strong in reality as the quality practitioners quite often do not have enough information to give this deterministic guess. In such a situation, we suggest to use an expected average time to signal EATS to evaluate the performance of the control chart, that is,

$$
E A T S=\int_{\Omega} A T S \times f_{\tau}(\tau) \mathrm{d} \tau
$$

where $f_{\tau}(\tau)$ is density function over the guessed support $\Omega$ of $\tau$. By using $E A T S$, it is not necessary to define any specific value of $\tau$. In the case there is no reference about the shape of $f_{\tau}(\tau)$, practitioners can choose a uniform distribution on the fixed support $\Omega=[a, b]$ for $\tau$. The new optimal triples $\left(K_{U}^{*}, H_{U}^{*}, h_{L}^{*}\right)$ and $\left(K_{D}^{*}, H_{D}^{*}, h_{L}^{*}\right)$ parameters combinations are found to minimize the out-of-control $E A T S_{1}$ given the in-control value $A T S_{0}$ and the constraint $E_{0}(h)=1$. In the calculation below, we consider two different ranges of shifts. The first range is $[0.5,1)$ corresponding to a decreasing shift and the second one is $(1,2]$ corresponding to an increasing shift. Table 5 presents the values of the EATS corresponding to the obtained optimal triples. The column entitled $F S I$ in this table is for the value of the $E A R L_{1}$, the control limit and the parameters $K_{U}$ or $K_{D}$ of FSI CUSUM- $\gamma^{2}$ charts.

The results in Table 5 show a similar trend on the effect of parameters $\tau, n, R_{U}\left(R_{D}\right)$ and $\gamma_{0}$ on the chart performance as for the deterministic shift size discussed above. Moreover, apart from the case $R_{U}\left(R_{D}\right)$ and $h_{S}$ are very small, say $R_{U}=0.05\left(R_{D}=0.05\right)$ and $h_{S}=0.1$ in this study, the values of the $E A T S_{1}$ and the other parameters in both the increase and decrease 
cases are the same. For example, when $n=10, \gamma_{0}=0.15, R=0.3, h_{S}=0.5$, we have $\left(K_{U}^{*}, H_{U}^{*}\right)=\left(K_{D}^{*}, H_{D}^{*}\right)=(0.17,3.31)$ and the same $\left(h_{L}^{*}, A T S_{1}\right)=$ $(1.17,14.5)$. This can be explained by the fact that the asymmetry of the distribution of the CV (see Figure 1) is compensated by the wider range of increasing shift size compared to the range of decreasing shift size (i.e. $(1,2]$ compared to $[0.5,1))$.

\section{Illustrative example}

In this section, using the data from a sintering process provided by an Italian company that manufactures sintered mechanical parts and presented by Castagliola et al. ${ }^{7}$, we present an implementation of the VSI CUSUM- $\gamma^{2}$ control chart. A pressure test drops time $T_{p d}$ from 2 bar to 1.5 bar larger than $30 \mathrm{~s}$ is assured to be seen as a quality characteristic related to the pore shrinkage. Throughout the sintering process, a molten copper is used to fill pores, allowing the drop time to be prolonged notably. In addition, the increase of quantity $Q_{C}$ of molten copper absorbed within the sintered compact during cooling leads to more the expected pressure drop time $T_{p d}$. It is stated that the preliminary regression study relating $T_{p d}$ to the quantity $Q_{C}$ of molten copper has demonstrated the presence of a constant proportionality $\sigma_{p d}=\gamma_{p d} \times \mu_{p d}$ between the standard deviation of the pressure drop time and its mean.

Suppose that the quality practitioner decided to monitor the CV $\gamma_{p d}=$ $\sigma_{p d} / \mu_{p d}$ in order to detect changes in the process variability. From the Phase I data, the in-control value of $\mathrm{CV}$ has been estimated, which is $\hat{\gamma}_{0}=0.417$. During Phase II, the dataset involving 20 new samples taken from the process after the occurrence of a special cause increasing process variability is presented in Table 6. We also assume an increase of the size $\tau=1.25$ in the coefficient of variation. From Equations (4) and (5), we get $\mu_{0}\left(\hat{\gamma}^{2}\right)=0.1557$, $\sigma_{0}\left(\hat{\gamma}^{2}\right)=0.1643$. The triple parameters $\left(K_{U}^{*}, H_{U}^{*}, h_{L}^{*}\right)$ of the upward VSI CUSUM- $\gamma^{2}$ chart which are optimal for detecting a shift from $\gamma_{0}=0.417$ to $\gamma_{1}=\gamma_{0} \times 1.25=0.521$ (an increase of $25 \%$ ) when $n=5$ and $h_{S}=0.1$ are found by the optimizing algorithm to be $(1.8181596,0.0831116,1.605802)$. As a result, we get $U C L^{+}=1.5644283$ and $U W L^{+}=0.0782214$.

Table 6 lists the values of $\hat{\gamma}_{i}^{2}$, the sampling interval of each sample, the total time covered by the sampling intervals and the statistic $C_{i}^{+}, i=1 . ., 20$. The statistic $C_{i}^{+}$is plotted in Figure 4 of the upward VSI CUSUM- $\gamma^{2}$ control chart. According to the observed result in the chart, several out-of-control situations are detected, corresponding to the boldfaced values in Table 6 . The process is considered to run out-of-control from the $13^{\text {th }}$ sample onward, confirming the occurrence of a special cause as it was expected by the 


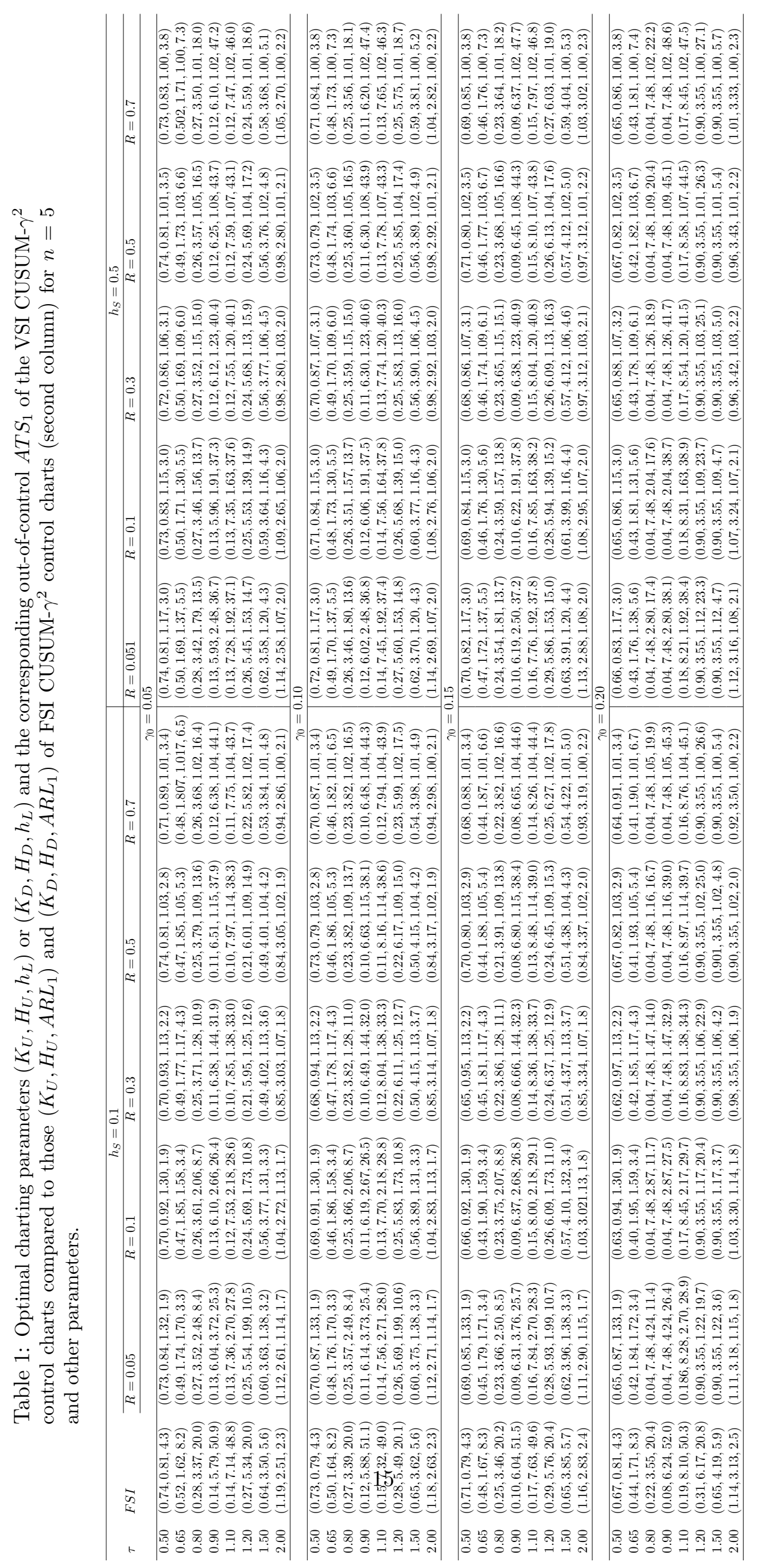




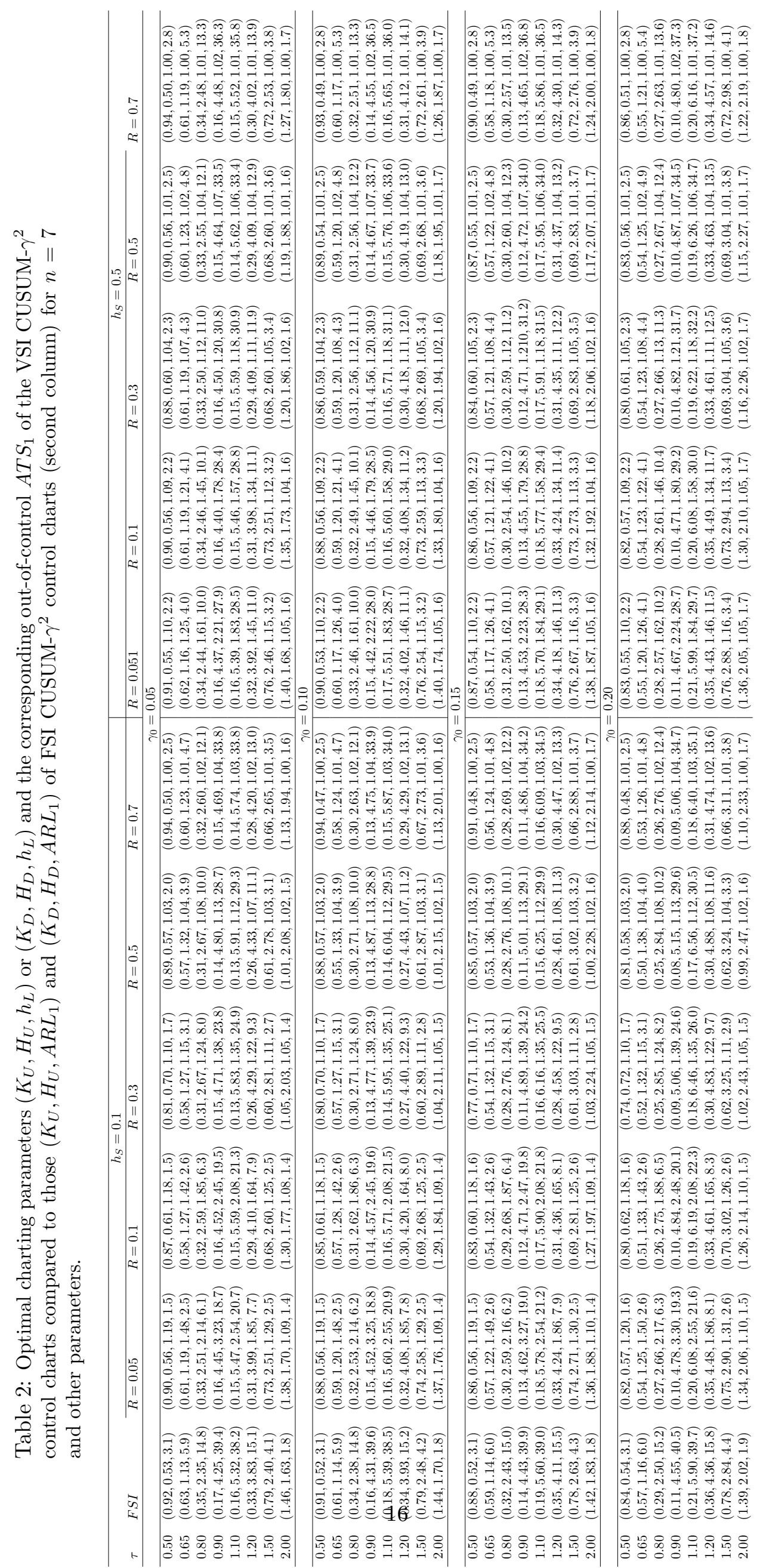




\begin{tabular}{|c|c|c|c|c|c|}
\hline & $\begin{array}{c}\tilde{0} \\
11 \\
\approx\end{array}$ & 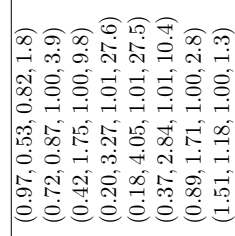 & 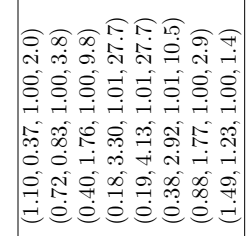 & 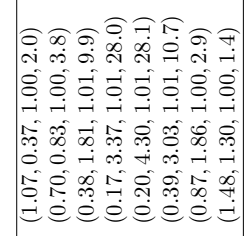 & 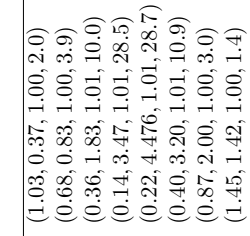 \\
\hline 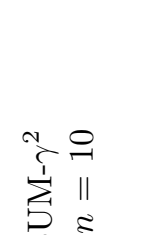 & 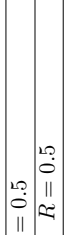 & 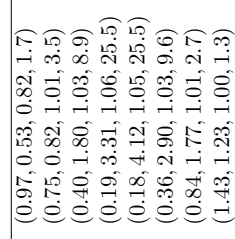 & 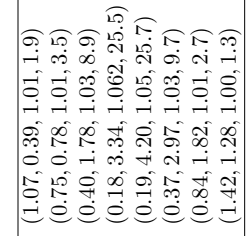 & 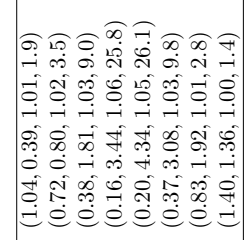 & 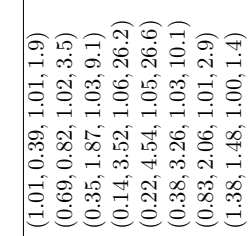 \\
\hline 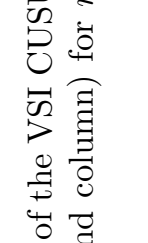 & $\left|\begin{array}{c}\infty \\
0 \\
i 1 \\
\approx\end{array}\right|$ & 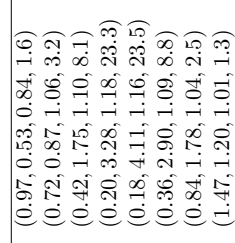 & 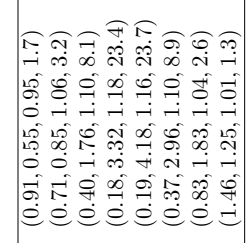 & 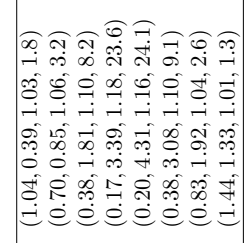 & 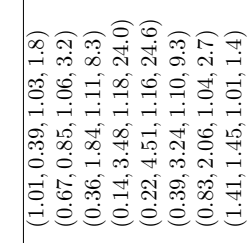 \\
\hline 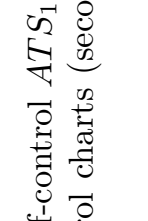 & & 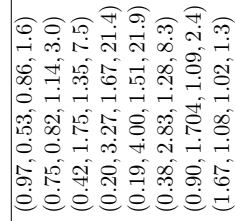 & 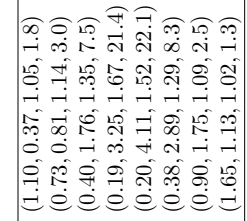 & 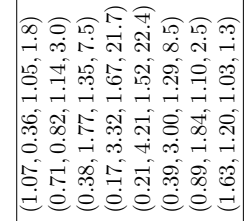 & $e^{10}$ \\
\hline 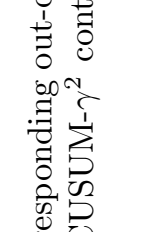 & 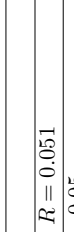 & 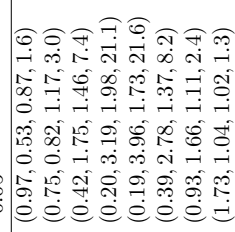 & 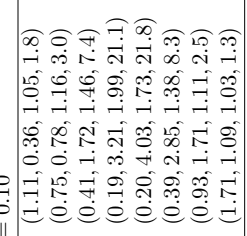 & 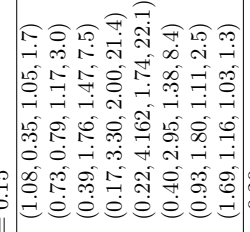 & 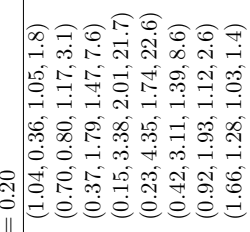 \\
\hline 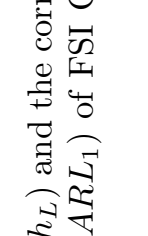 & {$\left[\begin{array}{l}1 \\
0 \\
11 \\
\approx\end{array}\right.$} & 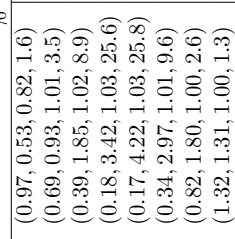 & 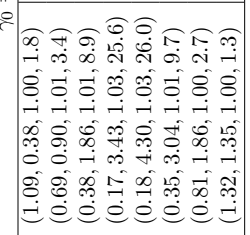 & 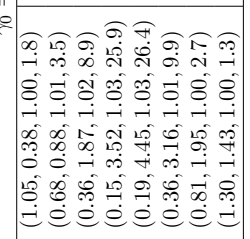 & $=\%$ \\
\hline 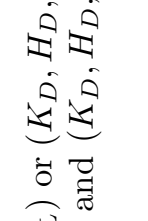 & {$\left[\begin{array}{l}2 \\
0 \\
1 \\
\approx\end{array}\right.$} & 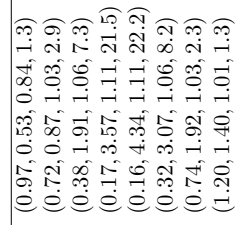 & 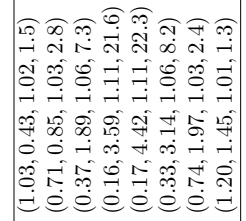 & 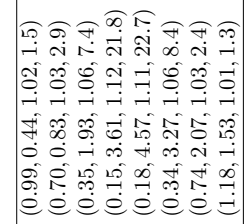 & $e e^{3}$ \\
\hline 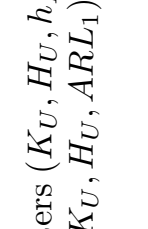 & & 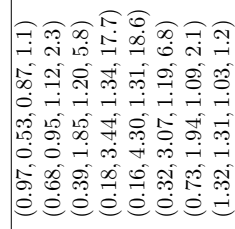 & 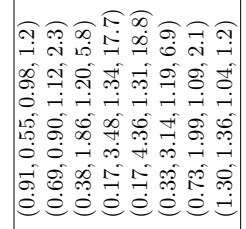 & 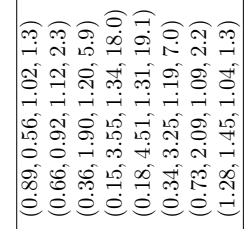 & $=5 \sqrt{6}$ \\
\hline 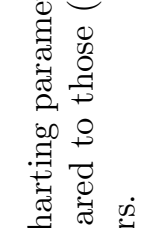 & $\left|\begin{array}{c}7 \\
0 \\
01 \\
\varepsilon\end{array}\right|$ & 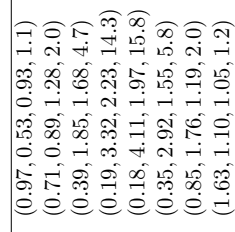 & 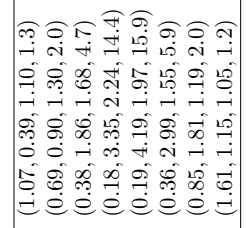 & 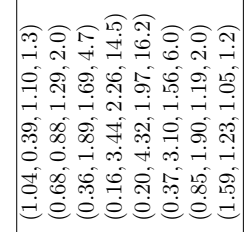 & \\
\hline 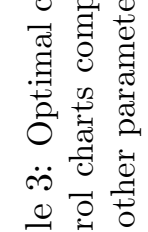 & $\mid \begin{array}{c}2 \\
0 \\
0 \\
11 \\
24\end{array}$ & 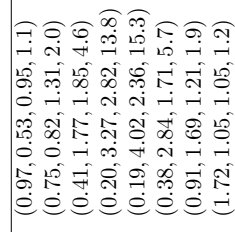 & 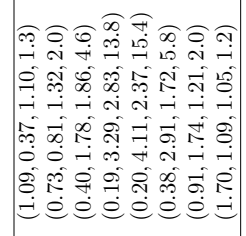 & 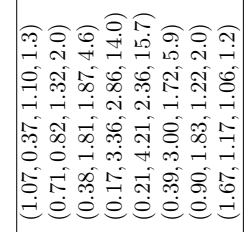 & \\
\hline & $\begin{array}{l}\bar{c} \\
\vec{L}\end{array}$ & 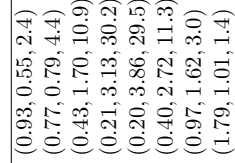 & 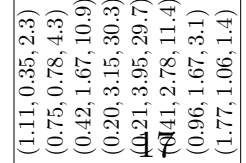 & 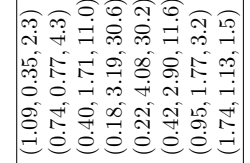 & \\
\hline & & 00000 & 10200000 & & \\
\hline
\end{tabular}




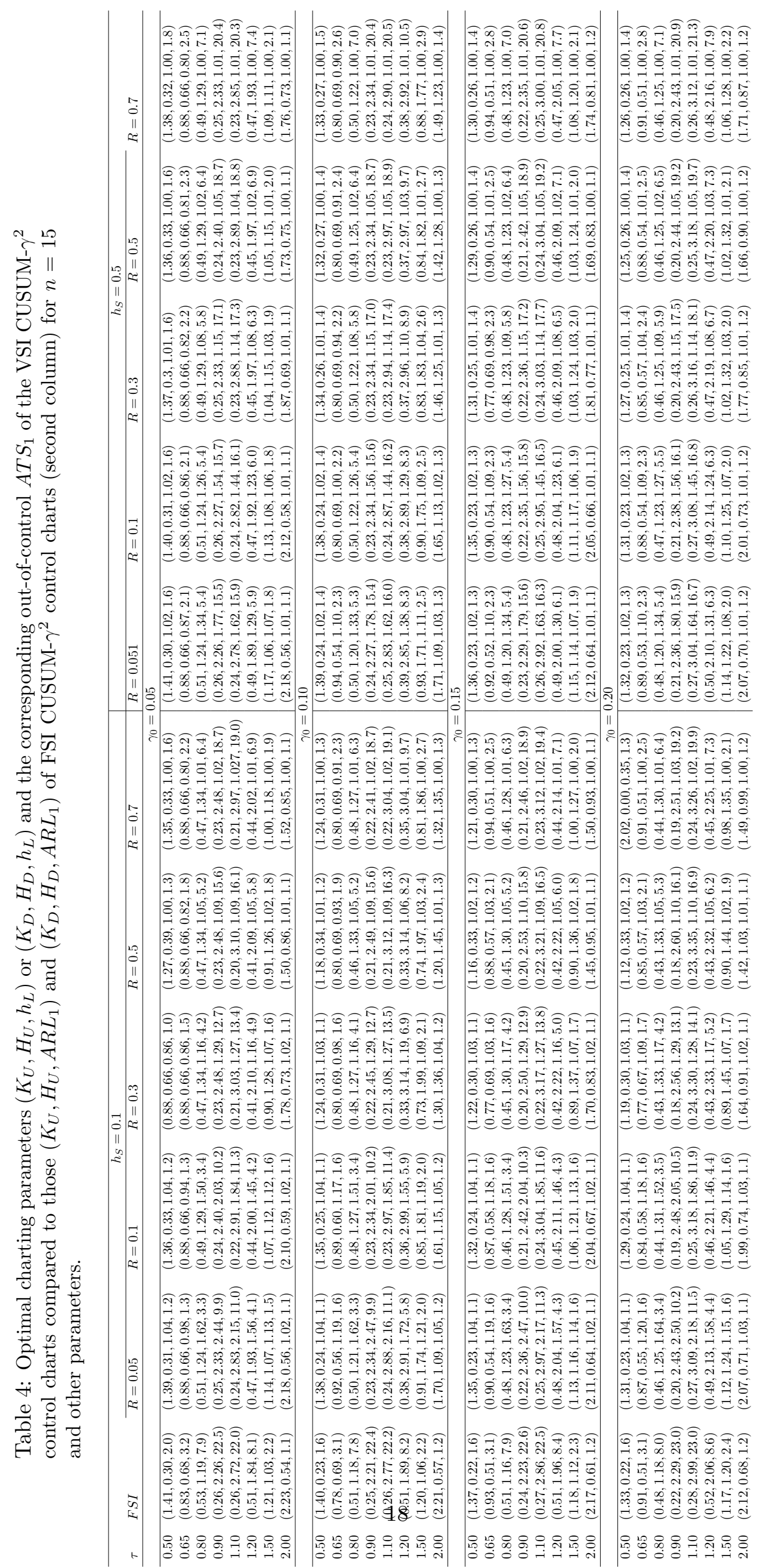




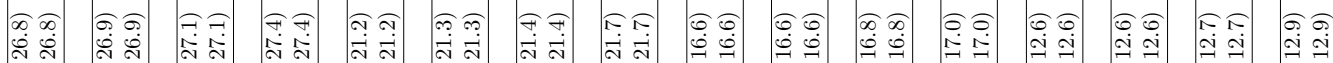
岛

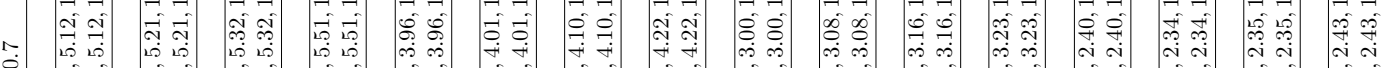

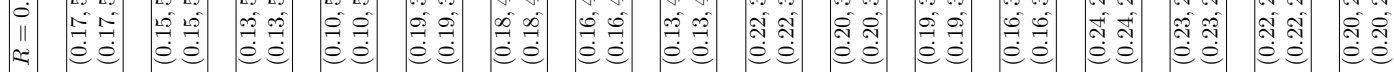

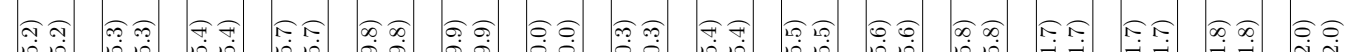

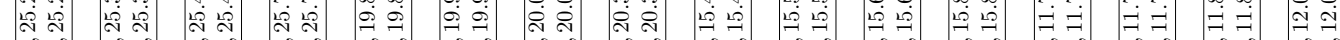

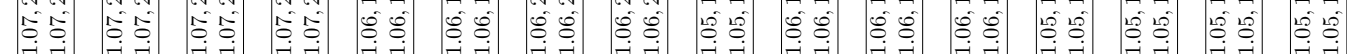

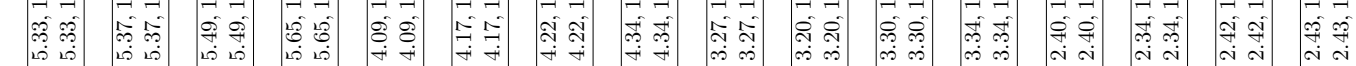

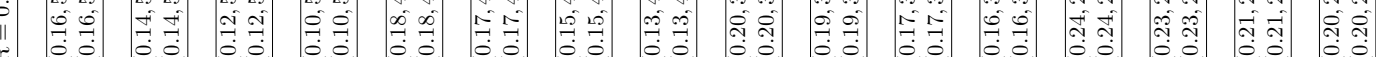

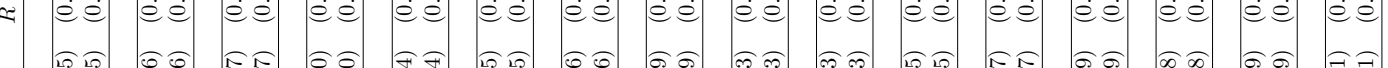

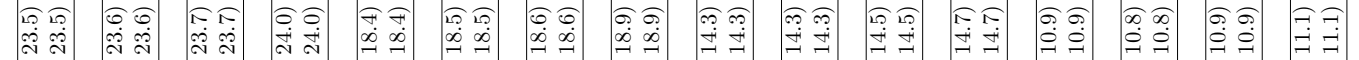

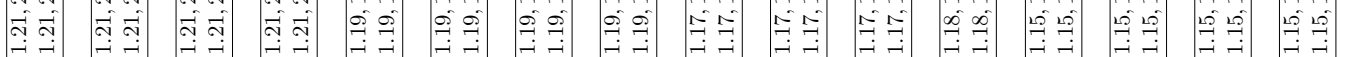

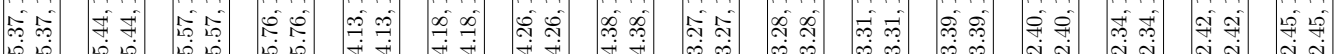

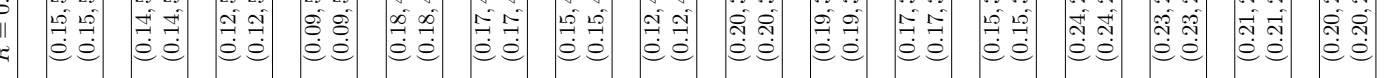

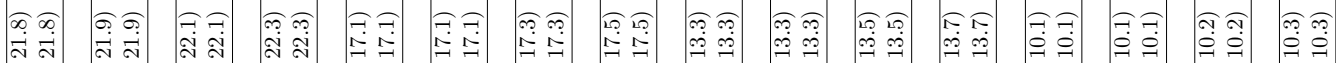

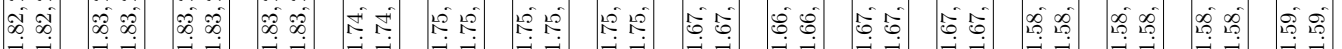

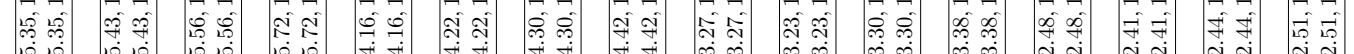

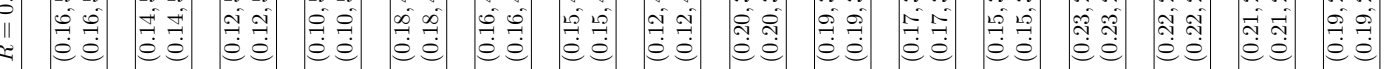

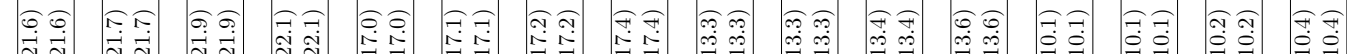

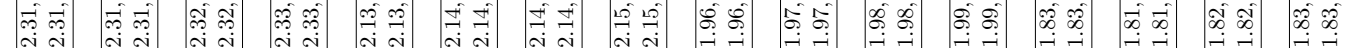
苗 I.

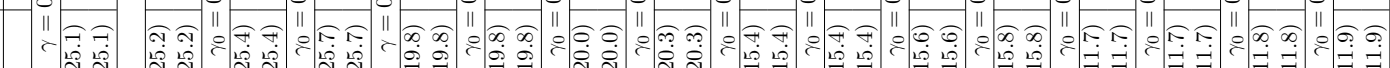
Th

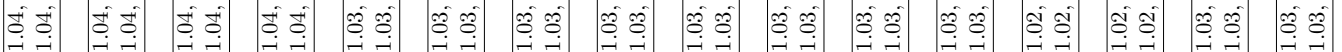

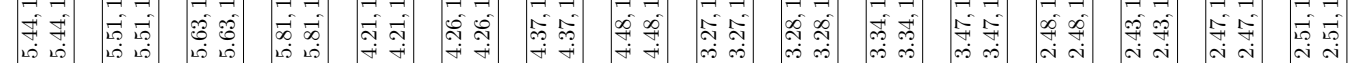

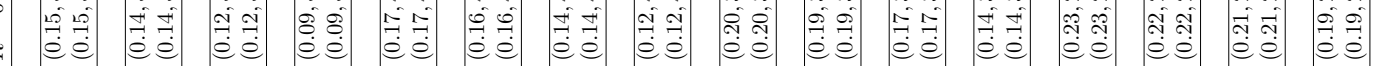
等

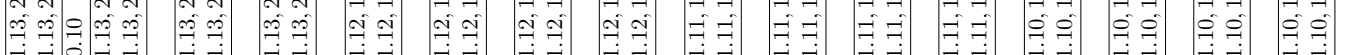

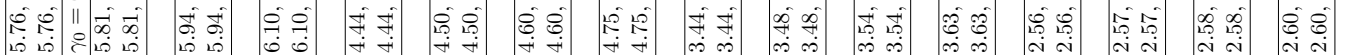

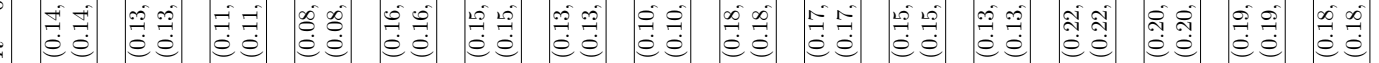
家家

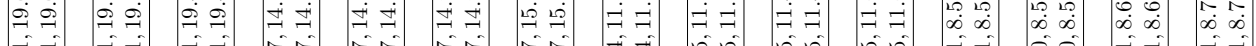

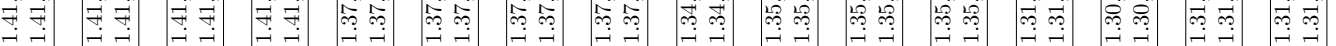

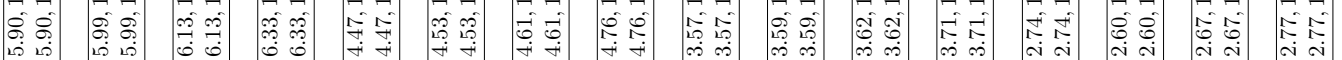

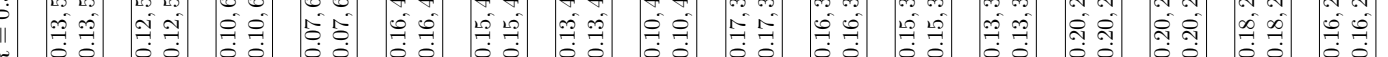

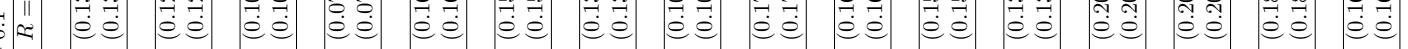

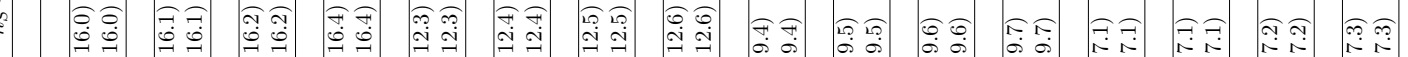

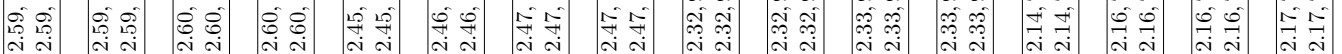

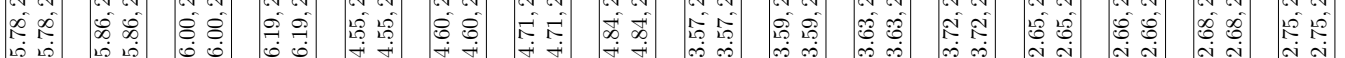

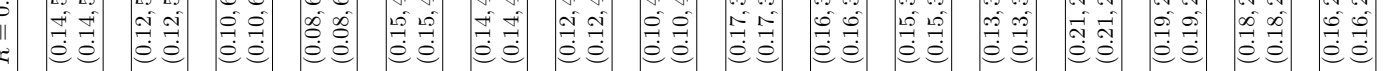

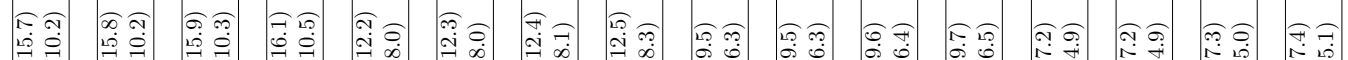

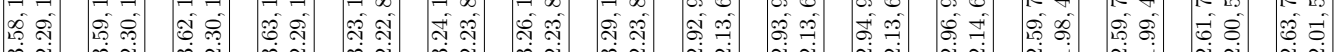

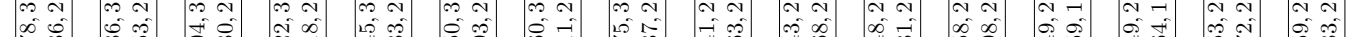

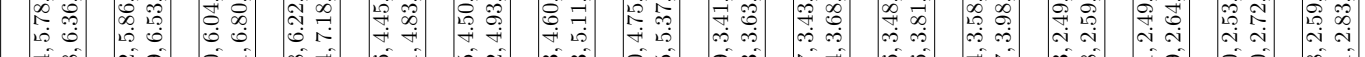

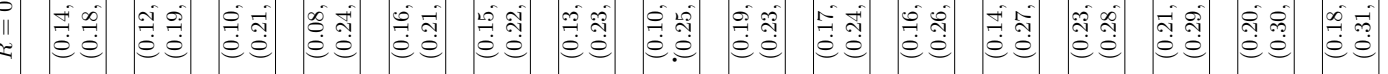

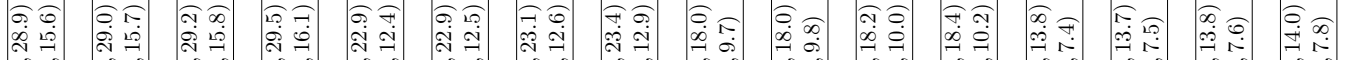

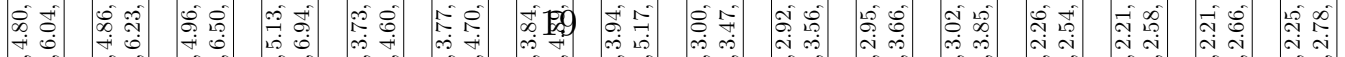
क

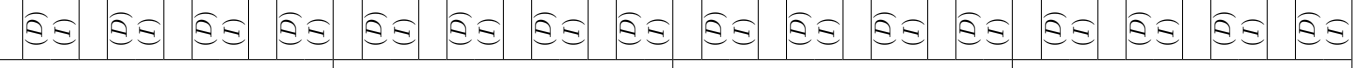


Phase II

\begin{tabular}{lclllll}
\hline$i$ & Sampling interval & Total time & $\bar{X}_{i}$ & $S_{i}$ & $\hat{\gamma}_{i}^{2}$ & $C_{i}^{+}$ \\
\hline 1 & 0.1 & 0.1 & 906.4 & 476.0 & 0.2756 & 0.039791 \\
2 & 1.61 & 1.71 & 805.1 & 493.9 & 0.3770 & 0.180981 \\
3 & 0.1 & 1.81 & 1187.2 & 1105.9 & 0.8686 & 0.813772 \\
4 & 0.1 & 1.91 & 663.4 & 304.8 & 0.2107 & 0.788662 \\
5 & 0.1 & 2.01 & 1012.1 & 367.4 & 0.1318 & 0.684653 \\
6 & 0.1 & 2.11 & 863.2 & 350.4 & 0.1648 & 0.613643 \\
7 & 0.1 & 2.21 & 1561.0 & 1652.2 & 1.1194 & 1.497234 \\
8 & 0.1 & 2.31 & 697.1 & 253.2 & 0.1318 & 1.393225 \\
9 & 0.1 & 2.41 & 1024.6 & 120.9 & 0.0139 & 1.171315 \\
10 & 0.1 & 2.51 & 355.3 & 235.2 & 0.4382 & 1.373706 \\
11 & 0.1 & 2.61 & 485.6 & 106.5 & 0.0480 & 1.185896 \\
12 & 0.1 & 2.71 & 1224.3 & 915.4 & 0.5595 & 1.509587 \\
13 & 0.1 & 2.81 & 1365.0 & 1051.6 & 0.5929 & $\mathbf{1 . 8 6 6 6 7 7}$ \\
14 & 0.1 & 2.91 & 704.0 & 449.7 & 0.4083 & $\mathbf{2 . 0 3 9 1 6 8}$ \\
15 & 0.1 & 3.01 & 1584.7 & 1050.8 & 0.4396 & $\mathbf{2 . 2 4 2 9 5 8}$ \\
16 & 0.1 & 3.11 & 1130.0 & 680.6 & 0.3624 & $\mathbf{2 . 3 6 9 5 4 9}$ \\
17 & 0.1 & 3.21 & 824.7 & 393.5 & 0.2275 & $\mathbf{2 . 3 6 1 2 4 0}$ \\
18 & 0.1 & 3.31 & 921.2 & 391.6 & 0.1806 & $\mathbf{2 . 3 0 6 0 3 0}$ \\
19 & 0.1 & 3.41 & 870.3 & 730.0 & 0.7039 & $\mathbf{2 . 7 7 4 1 2 1}$ \\
20 & 0.1 & 3.51 & 1068.3 & 150.8 & 0.0199 & $\mathbf{2 . 5 5 8 2 1 1}$ \\
\hline
\end{tabular}

Table 6: Phase II datasets from a sintering process in Castagliola et al. ${ }^{7}$

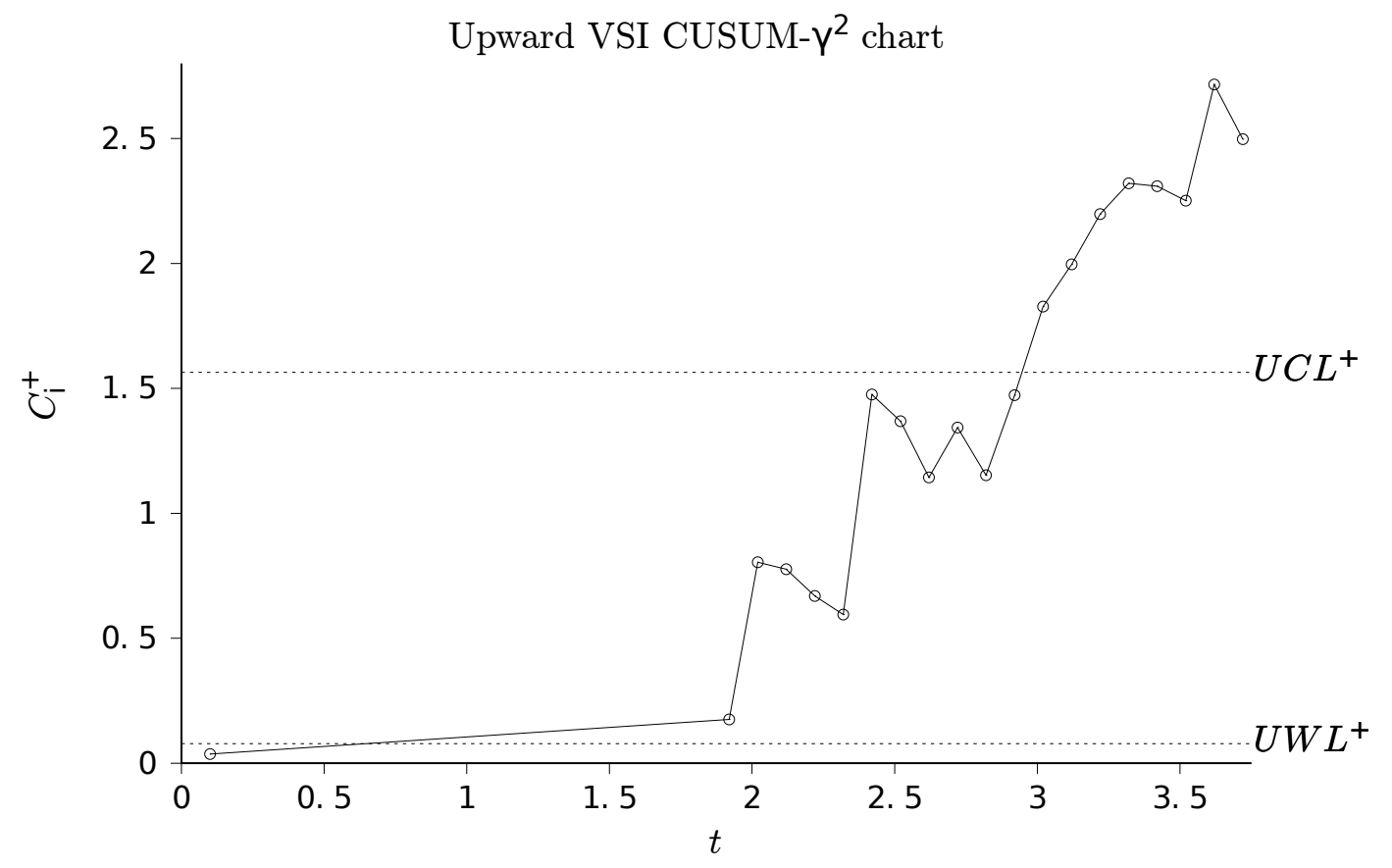

Figure 4: VSI CUSUM- $\gamma^{2}$ charts applied to the sintering process 
engineers.

\section{Concluding remarks}

We have investigated in this paper a new type of control chart to monitor the CV squared of a process. The statistical properties of VSI CUSUM- $\gamma^{2}$ are studied and their parameters are optimized for both fixed and random shift sizes. We have also introduced a new warning limit coefficient that makes the sampling interval in the safe region free to change provided that it optimizes the chart performance. A numerical comparison shows that the VSI CUSUM- $\gamma^{2}$ control charts outperform the standard CUSUM- $\gamma^{2}$ control charts in detecting assignable causes. The numerical results suggest a simple approach to improve the performance of the proposed charts by reducing the value of the warning limit coefficient and we recommend the use of 0.05 as an optimized value of this coefficient. Moreover, by designing two separately one-sided charts, our proposed charts are able to detect efficiently process shifts on both increase and decrease sides. This enables practitioners to be free to choose an adaptive type chart as a competitive chart to monitor downward shifts in the CV.

\section{References}

[1] Amdouni, A., Castagliola, P., Taleb, H. and Celano, G. 2015, 'Monitoring the coefficient of variation using a variable sample size control chart in short production runs', The International Journal of Advanced Manufacturing Technology 81(1-4), 1-14.

[2] Breunig, R. 2001, 'An almost unbiased estimator of the coefficient of variation', Economics Letters 70(1), 15-19.

[3] Brook, D. and Evans, D. 1972, 'An approach to the probability distribution of CUSUM run length', Biometrika 59(3), 539-549.

[4] Calzada, M. and Scariano, S. M. 2013, 'A synthetic control chart for the coefficient of variation', Journal of Statistical Computation and Simulation 83(5), 853-867.

[5] Castagliola, P., Achouri, A., Taleb, H., Celano, G. and Psarakis, S. 2013, 'Monitoring the coefficient of variation using a variable sampling interval control chart', Quality and Reliability Engineering International 29(8), 1135-1149.

[6] Castagliola, P., Achouri, A., Taleb, H., Celano, G. and Psarakis, S. 2015, 'Monitoring the coefficient of variation using a variable sample 
size control chart', The International Journal of Advanced Manufacturing Technology 81(9-12), 1561-1576.

[7] Castagliola, P., Celano, G. and Psarakis, S. 2011, 'Monitoring the coefficient of variation using EWMA charts', Journal of Quality Technology 43(3), 249-265.

[8] Iglewicz, B., Myers, R. H. and Howe, R. B. 1968, 'On the percentage points of the sample coefficient of variation', Biometrika 55(3), 580581.

[9] Kadri, F., Harrou, F., Chaabane, S., Sun, Y. and Tahon, C. 2016, 'Seasonal arma-based spc charts for anomaly detection: Application to emergency department systems', Neurocomputing 173, 2102-2114.

[10] Kang, C., Lee, M., Seong, Y. and Hawkins, D. 2007, 'A control chart for the coefficient of variation', Journal of Quality Technology 39(2), 151158.

[11] Khaw, K., Khoo, M., Yeong, W. and Wu, Z. 2017, 'Monitoring the coefficient of variation using a variable sample size and sampling interval control chart', Communications in Statistics - Simulation and Computation 46(7), 5722-5794.

[12] Münz, G. and Carle, G. 2008, Application of forecasting techniques and control charts for traffic anomaly detection, in 'Proceedings of the 19th ITC Specialist Seminar on Network Usage and Traffic, Berlin, Germany'.

[13] Reynolds, M. and Arnold, J. 1989, 'Optimal one-sided shewhart charts with variable sampling interval', Sequential Analysis 80(1), 181-192.

[14] Saccucci, M., Raid, W. and Lucas, J. 1992, 'Exponentially weighted moving average control schemes with variable sampling intervals', Communications in Statistics-Simulation and Computation 21(3), 627-657.

[15] Tran, P. H., Heuchenne, C. and Thomassey, S. August, 2021, An anomaly detection approach based on the combination of lstm autoencoder and isolation forest for multivariate time series data, in 'Proceedings of the 14th International FLINS Conference on Robotics and Artificial Intelligence (FLINS 2020), Cologne, Germany', World Scientific, pp. $18-21$.

[16] Tran, P. H. and Tran, K. P. 2016, 'The efficiency of CUSUM schemes for monitoring the coefficient of variation', Applied Stochastic Models in Business and Industry 32(6), 870-881. 
[17] Tran, P. H., Tran, K. P., Truong, T. H., Heuchenne, C., Nguyen, T. A. D. and Do, C. N. February, 2018, A variable sampling interval EWMA distribution-free control chart for monitoring services quality, in 'Proceedings of the 2018 International Conference on E-Business and Applications (ICEBA 2018), Da Nang, Vietnam', Da Nang, Vietnam, pp. 1-5.

[18] Yeong, W. C., Khoo, M. B. C., Teoh, L. K. and Rahim, M. A. 2017, 'Monitoring the coefficient of variation using a variable sampling interval EWMA chart', Journal of Quality Technology 49(3), 380-401.

[19] Yeong, W. C., Khoo, M. B. C., Teoh, W. L. and Castagliola, P. 2016, 'A control chart for the multivariate coefficient of variation', Quality and Reliability Engineering International 32(3), 1213-1225. 